\title{
Distribution of OXA-Type Class D $\beta$-Lactamase Genes Among Nosocomial Multi Drug Resistant Acinetobacter baumannii Isolated in Tehran Hospitals
}

\author{
Afsaneh Karmostaji ${ }^{1}$, Shahin Najar Peerayeh ${ }^{1,{ }^{*},}$, Ali Hatef Salmanian ${ }^{2}$ \\ ${ }^{1}$ Department of Bacteriology, Faculty of Medical Sciences, Tarbiat Modares University, Tehran, IR Iran \\ 2 National Institute of Genetic Engineering and Biotechnology, Tehran, IR Iran \\ ${ }^{*}$ Corresponding author: Shahin Najar Peerayeh, Department of Bacteriology, Faculty of Medical Sciences, Tarbiat Modares University, Tehran, IR Iran. Tel: +98-2182883870, Fax: +98- \\ 2182884555, E-mail: najarp_s@modares.ac.ir.
}

Received: September 17, 2012; Revised: January 19, 2013; Accepted: January 27, 2013

\begin{abstract}
Background:Multiple drug-resistant strains of Acinetobacter have become common in hospitals worldwide. The problem becomes more acute with increasing resistance to carbapenems, the last resort in the treatment of hospital acquired Acinetobacter baumannii infections. Objectives: The current study was conducted to determine the antimicrobial susceptibility patterns and prevalence of OXA-type carbapenemases, among clinical isolates of A. baumannii, in Tehran hospitals, Iran.

Materialsand Methods:Isolates were identified as A. baumannii by PCR with specific primersforbla OXA-51-like gene.Their susceptibilities to different antibiotics were determined using disk diffusion method. Isolates were then subjected to multiplex-PCR targeting bla oxa-51, bla oxa-24, blaoxa-23 and bla oxa-58 genes.

Results: Results showed that 123 of 131 (93.89\%) Acinetobacter species, possessed bla oxa-51-like gene and were identified as A.baumannii. $54.47 \%$ of isolates were resistant to amikacin, $67.47 \%$ resistant to imipenem and $84.55 \%$ resistant to meropenem.All isolates were susceptible to colistin and polymixin B. 43 of 123 A. baumannii isolates(34.95\%)were MDR. These isolates were resistant to amikacin, ciprofloxacin, imipenem, cefrazidim. Among 123 isolates, 100 (81.3\%) had an acquired oxa-23like carbapenemase 10 (8.1\%) possessed oxa-24-like, and 1 (0.81\%) possessed oxa-58-like carbapenemase.

Conclusions: The present study showed that bla OXA-23-like was the most frequent carbapenemase identified among carbapenemresistant A. baumannii isolated in Tehran hospitals. Evaluation of antibiotic resistance genes in A. baumannii, is necessary to control further dissemination of these antibiotic resistant genes.
\end{abstract}

Keywords: Beta-lactamases; Oxacillinase; Carbapenemase; Antibiotic Resistance

\section{Background}

Acinetobacter baumannii is a problematic nosocomial pathogen, especially in patients admitted to intensive care units (ICUs), those requiring mechanical ventilation, and patients with wound or burn injuries. This microorganism causes life threatening infections such as bacteremia, pneumonia, meningitis, urinary tract and wound infections (1). A. baumannii possess a remarkable ability to acquire plasmids, transposons, or integrons that carry clusters of resistant genes, and this ability leads to multi drug resistance (MDR). Control of MDR in A. baumannii is a medical concern, because of the limited therapeutic choices available. It should be noted that increasing resistance to carbapenems has been observed worldwide in the past decade.

Carbapenemase production is the most described mechanism of resistance to carbapenems (2). Carbapenem re- sistance in A. baumannii is mediated by the acquisition of a class B or a class D $\beta$-lactamase such as oxacillinase (3). Since the first description of a carbapenem-hydrolyzing oxacillinase, in 1993, several oxacillinases with a carbapenem-hydrolyzing activity have been reported (4). Nowadays, OXA-type carbapenemases have been divided into eight subgroups which four of them have been identified in A. baumannii: OXA-23-like consist of (OXA-23, OXA-27 and OXA-49); OXA-24-like (OXA-24, OXA-25, OXA-26, OXA40 and OXA-72); OXA-58; and OXA-51-like. The last group is a family of chromosomal enzymes typically found in A. baumannii. Acquired bla oxa-23 gene is located in transposon, mainly, Tn2006 (ISAba1 linked) and Tn2007 (ISAba4 linked). The strains which could produce Oxa-23, have been reported as sources of nosocomial outbreaks worldwide (2) In order to better understand and control multidrug-resistant $A$. baumannii, understanding the molecular basis of the infection is necessary. staff contact may have enhanced the spread of imipenem-resistant Acinetobacter sp. among different wards and different hospitals. Early recognition of the presence of imipenem-resistant genes, among Acinetobacter sp. is necessary in order to prevent their spread within the hospital environment. Copyright (c) 2013, Ahvaz Jundishapur University of Medical Sciences; Licensee Kowsar Ltd. This is an Open Access article distributed under the terms of the Creative Commons Attribution License (http://creativecommons.org/licenses/by/3.0), which permits unrestricted use, distribution, and reproduction in any medium, provided the original work is properly cited. 


\section{Objectives}

The current study described antimicrobial susceptibilities and conducted a multiplex PCR assay to detect alleles encoding oxacillinases. The study determines the prevalence of oxacillinase genes in clinical isolates of $\mathrm{A}$. baumannii from some Tehran hospitals.

\section{Materials and Methods}

\subsection{Bacterial Isolates}

Acinetobacter spp. $(\mathrm{n}=131)$ were isolated from $\mathrm{L}$ and $\mathrm{M}$, hospitals in Tehran, during 2010-2011. These bacteria were originally isolated from aspirated sputum, trachea, burn, wound and urinary tract infections. The clinical Acinetobacter isolates, were primarily identified by Gram staining as Gram negative coccobacillary rods that may initially appear in direct smears, as Gram positive cocci, non motile on S.I.M medium, oxidize negative and lack of lactose fermentation (5).

\subsection{DNA Extraction}

Genomic DNA was extracted by boiling method. Briefly, five to six colonies were suspended in $250 \mu \mathrm{l}$ sterile ultrapure water and boiled for 10 minutes. The samples were cooled on ice (10 minutes) and centrifuged at $14000 \mathrm{rpm}$ at room temperature.The supernatant was transferred to a new tube and kept at $4^{\circ} \mathrm{C}$ for further analysis (6).

\subsection{Detection of blaOXA-51-Like Gene to Identify A. baumannii Species}

All isolates were subjected to the PCR to detect blaoxa51-like gene which is unique to A. baumannii species $(7,8)$.

\subsection{MDR Definition}

Multidrug resistance was defined in this analysis as resistance to three or more representatives of the following classes of antibiotics: quinolones (ciprofloxacin), extended-spectrum cephalosporins (ceftazidime), aminoglycosides (amikacin, gentamicin), and carbapenems (imipenem, meropenem) (9).

\subsection{Susceptibility Testing}

Susceptibility to conventional antibiotics was performed by the disk diffusion method as recommended by the (CLSI). Colistin (10 $\mu \mathrm{g})$, Imipenem (10 $\mu \mathrm{g})$, Meropenem $(10 \mu \mathrm{g})$, Gentamicin $(10 \mu \mathrm{g})$, Ciprofloxacin $(5 \mu \mathrm{g})$, Amikacin $(30 \mu \mathrm{g})$, Cotrimoxazole $(25 \mu \mathrm{g})$, Cefepime (30 $\mu \mathrm{g})$, Cefotaxime $(30 \mu \mathrm{g})$, Aztreonam(30 $\mu \mathrm{g})$, Ceftazidime $(30 \mu \mathrm{g})$, and Polymyxin B (300 U) were obtained from Mast company (Pharmaceutical Inc. UK). Quality control was performed by testing the susceptibility of Escherichia coli ATCC 25922 (10).

\subsection{Detection of Carbapenem-Resistant Genes}

A multiplex polymerase chain reaction (PCR) assay was performed to detect the carbapenem-resistant genes in the A. baumannii isolates according to the method described by Woodford et al. ( 11,12 ). Primers were designed to amplify fragments of bla OXA-23, bla oxa-24,bla 0xa-58, carbapenemase genes. The amplification conditions were: initial denaturation at $94^{\circ} \mathrm{C}$ for 5 minutes, 30 cycles of $94^{\circ} \mathrm{C}$ for 25 seconds, $52^{\circ} \mathrm{C}$ for 40 seconds, $72^{\circ} \mathrm{C}$ for 50 seconds, and a final elongation at $72^{\circ} \mathrm{C}$ for 6 minutes (Table 1).

Table 1. Multiplex PCR Primers to Detect Genes Encoding oxa Carbapenemase

\begin{tabular}{|c|c|c|}
\hline Primers & Primer Sequence (5'-3') & $\begin{array}{l}\text { Product Size } \\
\text { (Base pair) }\end{array}$ \\
\hline OXA-51-like & $\begin{array}{l}\text { TAATGCTTTGATCGGCCTTGTG- } \\
\text { GATTGCACTTCATCTTGG }\end{array}$ & $353 \mathrm{bp}$ \\
\hline OXA-23-like & $\begin{array}{l}\text { GATCGGATTGGAGAAC- } \\
\text { CAGAATTTCTGACCGCATTTC- } \\
\text { CAT }\end{array}$ & $501 \mathrm{bp}$ \\
\hline OXA-24-like & $\begin{array}{l}\text { GGTTAGTTGGCCCCCTTA- } \\
\text { AAAGTTGAGCGAAAAGGGGATT }\end{array}$ & 246 bp \\
\hline OXA-58-like & $\begin{array}{l}\text { AAGTATTGGGGCTTGTGCT- } \\
\text { GCCCCTCTGCGCTCTACATAC }\end{array}$ & 599 bp \\
\hline
\end{tabular}

\section{Results}

\subsection{Strain Identification}

131 isolates of Acinetobacter, were identified by conventional identification methods. 91 (69.46\%) isolates were obtained in the intensive care unit, 21 (16.03\%) isolates in the internal wards, 10 (7.63\%) isolates in the Surgical ward, $5(3.8 \%)$ isolates in the emergency, $2(1.52 \%)$ isolates in ENT, and $2(1.52 \%)$ isolates, obtained in pediatrics. 7 (5.34\%) isolates were obtained from sputum. 16 (12.21\%) from burn, 65 (49.61\%) from pleural effusion, and 21 (16.03\%) isolates were obtained from wounds. In addition to them, there were isolates from urine, ear, and other samples. The blaOXA-51-like gene was amplified from genomic DNA to detect A.baumannii. 123 isolates (93.89\%) that gave a band for bla OXA-51-like gene, were identified as A. baumannii and $84(68.29 \%)$ of these isolates were found in ICU.

\subsection{Detection of Carbapenem-Resistant Genes}

Co-existence of different blaoxa genes among 123 isolates of A. baumannii and carbapeneme resistance of each isolate are indicated in Table 2. As observed in Table 2 , among the four isolates which contain only blaOXA51-like gene, no other oxacillinase genes, one isolate was carbapenem resistant. Along with other oxacillinase genes, increase in carbapenem resistance is observed. About bla OXA-23 gene, prevalence of gene and resistance 
rate is higher. None of the isolates carrying bla Oxa-58 associated with bla Oxa-23 or bla Oxa-24 genes. Table 3 shows the characteristics of isolates which possessed more than one of the carbapenemase genes. All of these isolates contain blaOXA-51-like gene, because this gene is intrinsic to all of A. baumannii isolates. These isolates have been isolated from two hospitals in Tehran named $\mathrm{L}$ and $M$. These isolates are resistant to three or more classes of antibiotics. According to the definition, it can be termed as $\operatorname{MDR}(9)$.

Table 2. Distribution of Different blaoxa Genes Among Clinical Isolates of Carbapenem Resistance A. baumannii

\begin{tabular}{llllll}
\hline Blaoxa Genes & No.(\%) & $\begin{array}{l}\text { Imipenem Resis- } \\
\text { tance Only, No. } \%)\end{array}$ & $\begin{array}{l}\text { Meropenem Resis- } \\
\text { tance Only, No. } \%)\end{array}$ & $\begin{array}{l}\text { Resistance to Both } \\
\text { Imipenem and Me- }\end{array}$ & $\begin{array}{l}\text { Total, Resistance } \\
\text { to Each of Car- } \\
\text { ropenem, No. (\%) } \\
\text { bapenem and to } \\
\text { Both of Carbapen- } \\
\text { ems, No. }(\%)\end{array}$ \\
\hline Oxa-51 only & & & & & $1(25 \%)$ \\
Oxa51 and Oxa-23 only & $100(81.3 \%)$ & $2(2 \%)$ & $0(0.0 \%)$ & $1(25 \%)$ & $89(89 \%)$ \\
Oxa-51 and Oxa-24 only & $10(8.13 \%)$ & $0(0.0 \%)$ & $5(50 \%)$ & $56(56 \%)$ & $8(80 \%)$ \\
Oxa-51 and Oxa-58 only & $1(0.81 \%)$ & $0(0.0 \%)$ & $1(100 \%)$ & $0(0.0 \%)$ & $1(100 \%)$ \\
$\begin{array}{l}\text { Oxa-51 and oxa-23 and } \\
\text { oxa-24 }\end{array}$ & $7(5.69 \%)$ & $0(0.0 \%)$ & $2(28.57 \%)$ & $3(42.8 \%)$ & $5(71.42 \%)$ \\
\hline
\end{tabular}

Table 3. Characteristics of Isolates Which Possessed More Than one of the Carbapenemase Genes

\begin{tabular}{|c|c|c|c|c|c|}
\hline Isolated Name & Hospital & Ward & Specimen & Antimicrobial Resistance & Oxa Genes \\
\hline $30 \mathrm{~T}$ & $\mathrm{~L}$ & medical & trachea & $\mathrm{AN}^{\mathrm{a}}, \mathrm{GM}^{\mathrm{a}}, \mathrm{SXT}^{\mathrm{a}}, \mathrm{CP}^{\mathrm{a}}, \mathrm{CAZ}^{\mathrm{a}}, \mathrm{CPM}^{\mathrm{a}}, \mathrm{CTX}^{\mathrm{a}}, \mathrm{ATM}^{\mathrm{a}}$ & oxa-23, оха- 4 , оха- 51 \\
\hline 47T & $\mathrm{L}$ & medical & sputum & MEN $^{\mathrm{a}}$, SXT, CP, CAZ, CPM, CTX, ATM & oxa- 24 , oxa- 23 , oxa- 51 \\
\hline $76 T$ & M & I.C.U & ear & AN, GM, MEN, SXT, CP, CAZ, CPM, CTX, ATM & oxa- 24, oxa- $23,0 \times a-51$ \\
\hline $77 \mathrm{~T}$ & M & I.C.U & trachea & AN, GM, IPM ${ }^{\mathrm{a}}$, MEN, SXT, CP, CAZ, CPM, CTX, ATM & oxa-23, oxa- 24, oxa-51 \\
\hline $114 T$ & M & I.C.U & burn & GM, SXT, CP, CAZ, CPM, CTX & oxa-23, oxa- 24, oxa- 51 \\
\hline 129T & $\mathrm{L}$ & I.C.U & trachea & GM, IPM, MEN, SXT, CP, CAZ, CPM, CTX, ATM, & oxa-23, oxa- 24, oxa-51 \\
\hline 131T & $\mathrm{L}$ & I.C.U & catheter & AN, GM, IPM, MEN, SXT, CP, CAZ, CPM, CTX, ATM & oxa-23, oxa- 24 , oxa-51 \\
\hline
\end{tabular}

a Abbreviations: IPM, Imipenem; MEN, Meropenem; GM, Gentamicin; CP, Ciprofloxacin; AN, Amikacin; SXT, Cotrimoxazole; CPM, Cefepime; CTX, Cefotaxime; AZT, Aztreonam; CAZ,Ceftazidime.

\subsection{Antimicrobial Susceptibilities}

Isolates of A. baumannii showed, 118 (95.93\%) to ciprofloxacin $95(77.23 \%)$ to gentamicin, 100 (81.3\%) to cefepime, 100 (97.56\%) to aztreonam, 117 (95.12\%) to ceftazidime 109 (88.61\%) resistance to trimeto prime sulphametoxazole, $67(54.47 \%)$ to amikacin, 83 (67.47\%) to imipenem, and 104 (84.55\%) to meropenem. All isolates were susceptible to colistin and Polymyxin B.43, of 123 A. baumannii isolates (34.95\%) were MDR. These isolates were resistant to amikacin, ciprofloxacin, imipenem, meropenem, ceftazidim. 37 (86.04\%) isolates of MDR A. baumannii possessed only bla oxa-23-like genes, and $2(4.65 \%)$ possessed both oxa24/oxa-23-like genes. One (2.35\%) possessed only bla oxa51-likegene (Figure 1).

\section{Discussion}

A . baumannii is considered as one of the most important nosocomial pathogens. The occurrence of MDR and pan drug-resistant A. baumannii is a growing concern. The current study indicated that, 43 of 123 A. baumannii
Figure 1. Detection of Genes Encoding oxa Carbapenemase by Multiplex PCR

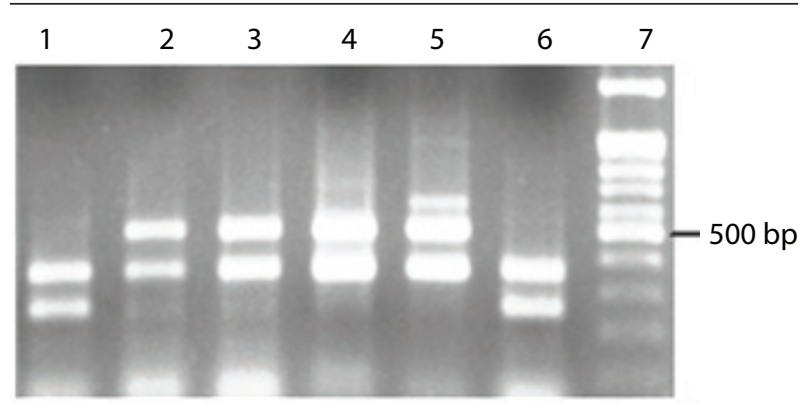

Line 1 and 6, oxa-51-like gene (353 bp) and oxa-24-like gene (246 bp), Line 2-4, oxa-51-like gene (353 bp) and oxa-23-like gene (501 bp), Line 5, oxa-51like gene (353 bp) and oxa-23-like gene (501 bp) and oxa-58-like gene (599 bp), Lane 7,100 bp DNA ladder

isolates (34.95\%) were MDR. These isolates were resistant to amikacin, ciprofloxine, imipenem, meropenem, ceftazidim. As Table 3 shows, 5 of 7 (71.43\%) isolates which 
carried more than two oxacillinase genes, and were MDR, had been isolated from intensive care units. Resistance rates can differ according to the country, hospital under review, and depend on biological, epidemiological or methodical factors (13). In 1999, 15 hospitals in Brooklyn, reported high rates of MDRA. baumannii infection, twelve percent of the strains were resistant to all commonly used drugs (14). During the years 2003 to 2004, of A. baumannii isolates, $76 \%$ were multi-drug resistant (MDR); almost half of them being resistant to every tested antimicrobial except, imipenem (15).

In Washington DC in 2006, 89\% of Acinetobacter isolates, were resistant to at least 3 drugs, meeting the criteria for multidrug resistance (16). The high rate of MDR isolates in studies referred to here between 2003 to 2006, compared to the MDR rate obtained in the current study is due to the fact that most isolates in those studies, were from admitted military personnel during Iraq and Afghanistan wars. This shows that, an increase in the use of broad-spectrum antibiotics along with war and natural disasters contribute to the isolation of more antimicrobial resistant bacteria. The current study also, describes the important role of class D carbapenem hydrolyzing $\beta$-lactamases, and in particular blaoxa-23-like gene, in the dissemination of imipenem resistant A. baumannii isolates in Tehran hospitals. 56\% of A. baumannii isolates which possessed bla oxa-23-like genes, were resistant to both imipenem and meropenem, and 37 (86.04\%) isolates of MDR A. baumannii possessed only bla oxa-23-like genes. Overall rate of resistance to imipenem was $83(67.47 \%)$ and to meropenem 104 (84.55\%).

Several reports from around the world indicate a large increase in the rates of carbapenem-resistant A. baumannii from $8 \%$ in 2003 to $52 \%$ and $74 \%$ in 2005 and finally to $96 \%$ in $2007(17,18)$. In Iran it was reported as $49.3 \%$ resistance to imipenem and 50\% resistance to meropenem in 2008 (12), 52.5\% resistance to imipenem and meropenem in 2009 (19), and $49.26 \%$ resistance to imipenem in 2011 (20). Distribution of blaoxa alleles among Acinetobacter isolates, in Tehran was as follow: blaoxa-23-like / bla oxa-51 like was detected in $25 \%$, blaoxa-24-like / bla oxa-51-like in $17.9 \%$ and blaoxa-58-like / bla oxa-51-like was detected in $9 \%$ of the isolates in 2008 (12). Bla oxa-23-like in $25 \%$, bla oxa-58 -like in $21.2 \%$ and bla oxa-24-like in $15 \%$ were detected in $2009(12,19)$.

In other studies, increasing level of blaoxa-23 was reported so that, $94 \%$ and $84 \%$ of the isolates were positive for bla oxa-51 and bla oxa-23 like genes in 2011 (20). $88.7 \%$ bla OXA-23-like, 1.6\% bla OXA-40-like, and 3.2\% had bla OXA-58-like resistance genes in 2012 in North West of Iran (21). The current study report in 2010-2011 also demonstrates an increased prevalence of bla oxa-23-like gene (81.3\%), but different data about blaoxa-24-like (8.13\%) and bla oxa-58 genes (0.81\%). Explanation of this difference is that in one of the previous studies (12), all isolates of Acinetobacter (A. baumannii and NON-A. baumannii), sensitive and resistant strains were included. A part of this phenomenon may be due to the fact that different hospitals were evaluated.

It is expected that different hospitals present different molecular epidemiology of carbapenem-resistant. Studies in various parts of the world revealed considerable geographical differences in the types of class D carbapenem hydrolyzing $\beta$-lactamases (18). In Taiwan, in 2006, 45\% of $A$. baumannii isolates were resistant or intermediate to imipenem and meropenem. However, they found only one bla OXA-23 and one bla OXA-24 harboring A. baumannii isolate (22). Similar observations have been reported in other Taiwanese studies $(23,24)$. In other countries, the widespread dissemination of carbapenem -resistant Acinetobacter spp. with bla oxa-23-like or bla oxa-24-like has been reported.

Mendes et al., during 2006 - 2007, from 41 medical centers located in 10 countries, reported the class D carbapenemase genes in $70 \%$ of the strains. Bla oxa-23-like was the most common gene, which accounted for $95.0 \%$ of the class D carbapenemase-encoding genes detected, followed by a lower occurrence of bla oxa-58 (11.9\%) and bla 0xa-24/40 $(5.6 \%)(18,25)$. In Ohio, the United States in $2009,13 \%$ of imipenem resistant isolates, contained the blaoxa-23.The other class D carbapenemase, including bla oxa-24 and bla oxa-58 like genes could not be identified (2). In Bulgaria, $72.72 \%$ of carbapenem-resistant isolates were positive for bla oxa-23-like and $27.27 \%$ were positive for bla oxa-58-like (17).

The current study found four strains that contained only the bla oxa-51-like. Three of these isolates were sensitive to imipenem and meropenem. Therefore it implies that the relationship between bla oxa-51-like and resistance of A. baumannii to carbapenem is dependent on other factors such as the presence of ISAba1-bla oxa-51like that play an important role, as a 'mobile promoter. It is suggested that the presence of ISAba1-blaoxa-51-like be examined. In addition, more studies are needed to determine the clonal relatedness to know if dissemination of the blaoxa genes results in strains that are derived from a common ancestor or the result of strains exchanging a transposable genetic element. It is also possible that the MDR strains which circulate in hospitals are distinct lineages or groups of lineages within A. baumannii, which suggests that the problem of resistance might be associated with a few numbers of $A$. baumannii lineages.

The current study showed low susceptibility rates to most of the available antimicrobial agents for treatment of infections caused by A. baumannii, except for polymyxin $B$ and colistin, while other studies in Iran have demonstrated $12 \%$ resistance to colistin and 3\% resistance to polymixin B in 2011 (20),and 8.8\% resistance to polymixin B in 2009 (19), multi-drug strains resistant even to colistin suggests, we should be looking for novel Therapeutic strategies. It should be noted that, fight against MDR A. baumannii (and other MDR organisms) is far beyond the hospital and needs a common strategy of decision makers and health-care officials, the challenge being to make 
hospitals as a safe place for patients.

\section{Acknowledgements}

The study is supported by Tarbiat Modares University.

\section{Authors' Contribution}

None Declared.

\section{Financial Disclosure}

None Declared.

\section{Funding/Support}

None Declared.

\section{References}

1. Towner KJ. Acinetobacter: an old friend, but a new enemy. J Hosp Infect. 2009;73(4):355-63.

2. Srinivasan VB, Rajamohan G, Pancholi P, Stevenson K, Tadesse D, Patchanee P, et al. Genetic relatedness and molecular characterization of multidrug resistant Acinetobacter baumannii isolated in central Ohio, USA. Ann Clin Microbiol Antimicrob. 2009;8:21.

3. Lin MF, Chang KC, Lan CY, Chou J, Kuo JW, Chang CK, et al. Molecular epidemiology and antimicrobial resistance determinants of multidrug-resistant Acinetobacter baumannii in five proximal hospitals in Taiwan. Jpn J Infect Dis. 2011;64(3):222-7.

4. Heritier C, Poirel L, Fournier PE, Claverie JM, Raoult D, Nordmann P. Characterization of the naturally occurring oxacillinase of Acinetobacter baumannii. Antimicrob Agents Chemother. 2005;49(10):4174-9.

5. Murray M. Manual of Clinical Microbiology. 8 ed. Washington DC, USA: ASM Press; 2003. p. 749-52.

6. Andriamanantena TS, Ratsima E, Rakotonirina HC, Randrianirina F, Ramparany L, Carod JF, et al. Dissemination of multidrug resistant Acinetobacter baumannii in various hospitals of Antananarivo Madagascar. Ann Clin Microbiol Antimicrob. 2010;9:17.

7. Turton JF, Woodford N, Glover J, Yarde S, Kaufmann ME, Pitt TL. Identification of Acinetobacter baumannii by detection of the blaOXA-51-like carbapenemase gene intrinsic to this species. $J$ Clin Microbiol. 2006;44(8):2974-6.

8. Alsultan AA, Hamouda A, Evans BA, Amyes SG. Acinetobacter baumannii: emergence of four strains with novel bla(OXA51-like) genes in patients with diabetes mellitus. $J$ Chemother. 2009;21(3):290-5.

9. Magiorakos AP, Srinivasan A, Carey RB, Carmeli Y, Falagas ME, Giske CG, et al. Multidrug-resistant, extensively drug-resistant and pandrug-resistant bacteria: an international expert proposal for interim standard definitions for acquired resistance. Clin Microbiol Infect. 2012;18(3):268-81.

10. Lee K, Yum JH, Yong D, Lee HM, Kim HD, Docquier JD, et al. Novel acquired metallo-beta-lactamase gene, bla(SIM-1), in a class 1 integron from Acinetobacter baumannii clinical isolates from Korea. Antimicrob Agents Chemother. 2005;49(11):4485-91.

11. Woodford N, Ellington MJ, Coelho JM, Turton JF, Ward ME, Brown S, et al. Multiplex PCR for genes encoding prevalent OXA carbapenemases in Acinetobacter spp. Int J Antimicrob Agents. 2006;27(4):351-3.

12. Feizabadi MM, Fathollahzadeh B, Taherikalani M, Rasoolinejad M, Sadeghifard N, Aligholi M, et al. Antimicrobial susceptibility patterns and distribution of blaOXA genes among Acinetobacter spp. Isolated from patients at Tehran hospitals. Jpn J Infect Dis. 2008;61(4):274-8.

13. Dijkshoorn L, Nemec A, Seifert H. An increasing threat in hospitals: multidrug-resistant Acinetobacter baumannii. Nat Rev Microbiol. 2007;5(12):939-51.

14. Landman D, Quale JM, Mayorga D, Adedeji A, Vangala K, Ravishankar J, et al. Citywide clonal outbreak of multiresistant Acinetobacter baumannii and Pseudomonas aeruginosa in Brooklyn, NY: the preantibiotic era has returned. Arch Intern Med. 2002;162(13):1515-20.

15. Davis KA, Moran KA, McAllister CK, Gray PJ. Multidrug-resistant Acinetobacter extremity infections in soldiers. Emerg Infect Dis. 2005;11(8):1218-24.

16. Hujer KM, Hujer AM, Hulten EA, Bajaksouzian S, Adams JM, Donskey CJ, et al. Analysis of antibiotic resistance genes in multidrugresistant Acinetobacter sp. isolates from military and civilian patients treated at the Walter Reed Army Medical Center. Antimicrob Agents Chemother. 2006;50(12):4114-23.

17. Stoeva T, Higgins PG, Savov E, Markovska R, Mitov I, Seifert H. Nosocomial spread of OXA-23 and OXA-58 beta-lactamase-producing Acinetobacter baumannii in a Bulgarian hospital. J Antimicrob Chemother. 2009;63(3):618-20.

18. Qi C, Malczynski M, Parker M, Scheetz MH. Characterization of genetic diversity of carbapenem-resistant Acinetobacter baumannii clinical strains collected from 2004 to 2007. J Clin Microbiol. 2008;46(3):1106-9.

19. Taherikalani M, Fatolahzadeh B, Emaneini M, Soroush S, Feizabadi MM. Distribution of different carbapenem resistant clones of Acinetobacter baumannii in Tehran hospitals. New Microbiol. 2009;32(3):265-71.

20. Shahcheraghi F, Abbasalipour M, Feizabadi M, Ebrahimipour $\mathrm{G}$, Akbari N. Isolation and genetic characterization of metallobeta-lactamase and carbapenamase producing strains of Acinetobacter baumannii from patients at Tehran hospitals. Iran J Microbiol. 2011;3(2):68-74.

21. Sohrabi N, Farajnia S, Akhi MT, Nahaei MR, Naghili B, Peymani A, et al. Prevalence of OXA-type beta-lactamases among Acinetobacter baumannii isolates from Northwest of Iran. Microb Drug Resist. 2012;18(4):385-9.

22. Lin YC, Hsia KC, Chen YC, Sheng WH, Chang SC, Liao MH, et al. Genetic basis of multidrug resistance in Acinetobacter clinical isolates in Taiwan. Antimicrob Agents Chemother. 2010;54(5):2078-84.

23. Lee YT, Huang LY, Chiang DH, Chen CP, Chen TL, Wang FD, et al. Differences in phenotypic and genotypic characteristics among imipenem-non-susceptible Acinetobacter isolates belonging to different genomic species in Taiwan. Int J Antimicrob Agents. 2009;34(6):580-4.

24. Lu PL, Doumith M, Livermore DM, Chen TP, Woodford N. Diversity of carbapenem resistance mechanisms in Acinetobacter baumannii from a Taiwan hospital: spread of plasmid-borne OXA-72 carbapenemase. J Antimicrob Chemother. 2009;63(4):641-7.

25. Mendes RE, Bell JM, Turnidge JD, Castanheira M, Jones RN. Emergence and widespread dissemination of OXA-23, -24/40 and -58 carbapenemases among Acinetobacter spp. in Asia-Pacific nations: report from the SENTRY Surveillance Program. J Antimicrob Chemother. 2009;63(1):55-9.

Please cite this paper as: Karmostaji A, Najar Peerayeh SH, Hatef Salmanian A. Distribution of OXA-Type Class D $\beta$-Lactamase Genes Among Nosocomial Multi Drug Resistant Acinetobacterbaumannii Isolated in Tehran Hospitals. Jundishapur J Microbiol. 2013; 6(5): e8219. DOI:10.5812/ijem.8219 